\title{
A Survey on Geographical Routing Strategies in VANET
}

\author{
P. G. Nirmal \\ Research Scholar, CSE \\ Department \\ G. H. Raisoni College of \\ Engineering, Nagpur, India.
}

\author{
A. R. Deshmukh \\ Research Scholar, ETC \\ Department \\ G. H. Raisoni College of \\ Engineering, Nagpur, India.
}

\author{
S. S. Dorle \\ H.O.D, ETRX Department \\ G. H. Raisoni College of \\ Engineering, Nagpur, India.
}

\begin{abstract}
Vehicular Ad Hoc Network is an emerging technology nowadays which plays vital role in case of vehicular communication. It allows vehicles on the road to form network and communicate with each other without aid of any infrastructure. As VANET is highly dynamic and infrastructure less network thus, it requires efficient routing protocol to route packet. Position based routing protocols like GPSR, GPCR, CAR are more suitable for VANET and are widely used. In this paper survey on different position based routing protocols has been done .The main goal of this paper is, to provide detailed classification and overview of various position based routing protocols in VANET.
\end{abstract}

\section{Keywords}

VANET, Position based routing protocols, Node, DTN, Packet

\section{INTRODUCTION}

Vehicular Ad Hoc Network (VANET) is a subclass of Mobile Ad Hoc Network (MANET) [1] where, vehicle act as a mobile nodes. VANETs are highly dynamic in nature because of frequent topology changes due to high speed and movement of vehicles. This makes routing a very challenging issue in case of VANET .In order to resolve this issue, various type of routing protocols have been developed. The main objective of routing protocols in ad hoc network is to create optimal path between source and destination. In addition to this, routing protocols tend to reduce amount of overhead, bandwidth utilization and delay. There are various types of routing protocols available for VANET. The routing protocols in VANET are classified as topology based routing, cluster based routing, broadcast routing, geo cast routing and position based routing. This paper deals with position based routing protocols in VANET.

\section{POSITION BASED ROUTING PROTOCOL}

In position based routing protocol, each and every node or vehicle in the network must know its own and neighbor node's geographical position [2] .Nodes make use of GPS to find their location .Position based routing protocol do not maintain routing table or do not exchange link state information thus routing overhead is reduced. The position based routing protocols are classified as Delay Tolerant Network (DTN), Non Delay Tolerant Network (Non-DTN) and Hybrid. DTN routing protocol do not consider discontinuous connectivity. Non-DTN routing protocol consider discontinuous connectivity whereas Hybrid is a combination of DTN and Non-DTN routing protocol. Detailed classification of position based routing protocol is as shown in figure1. All these position based routing protocols are discussed in the following sections.

\section{DELAY TOLERANT NETWORK(DTN)}

In DTN ,carry and forward strategy is used. This strategy is very useful in the case of frequent disconnections of the nodes.

\subsection{Vehicle Assisted Data Delivery (VADD)}

This protocol is proposed by Jing Zhao and Guohong Cao. The main objective of VADD [3] is to forward packet to best path with minimum delay in data delivery. Carry and forward strategy is used by VADD .Sometimes it is necessary to execute dynamic path selection in packet forwarding process because of frequently changing topology, thus packet delivery along the pre computed path could not be assured. It has three modes of operation namely intersection, straightway and destination .Optimal path for forwarding packet is selected through these modes. VADD protocol has several ways to choose next node for forwarding packet. The first one is LVADD i.e. location first probe in which closest node to target intersection is selected without knowing travelling direction. Second one is D-VADD i.e. direction first probe in which nodes having same direction are selected.

\subsection{Scalable Knowledge based Vehicular Routing ( SKVR)}

SKVR works by dividing network into two domains, inter domain and intra domain [4] . In inter domain routing, source and targeted locations are on different routes where as in intra domain routing they are on same routes. In case of interdomain routing, on reaching destination domain message is sent to vehicle which is in targeted location domain. Intra domain routing is used in case of intra domain routing, depending on entries of contact list ,messages are sent in forward or reverse directions. If there is no vehicle in sending vehicle's contact list of targeted domain then messages are delivered to other vehicle in contact list.

\subsection{Geographical Opportunistic (GeOpps)}

In this protocol, [5] in order to send messages to a particular geographical location, GPS information is used. To choose vehicle that carry the information, information from vehicle's GPS is utilized which gives proper routes. Then neighbor vehicle computes nearest point to destination through suggested routes. If vehicle can send packet quickly to its destination then it is marked as next packet carrier. 


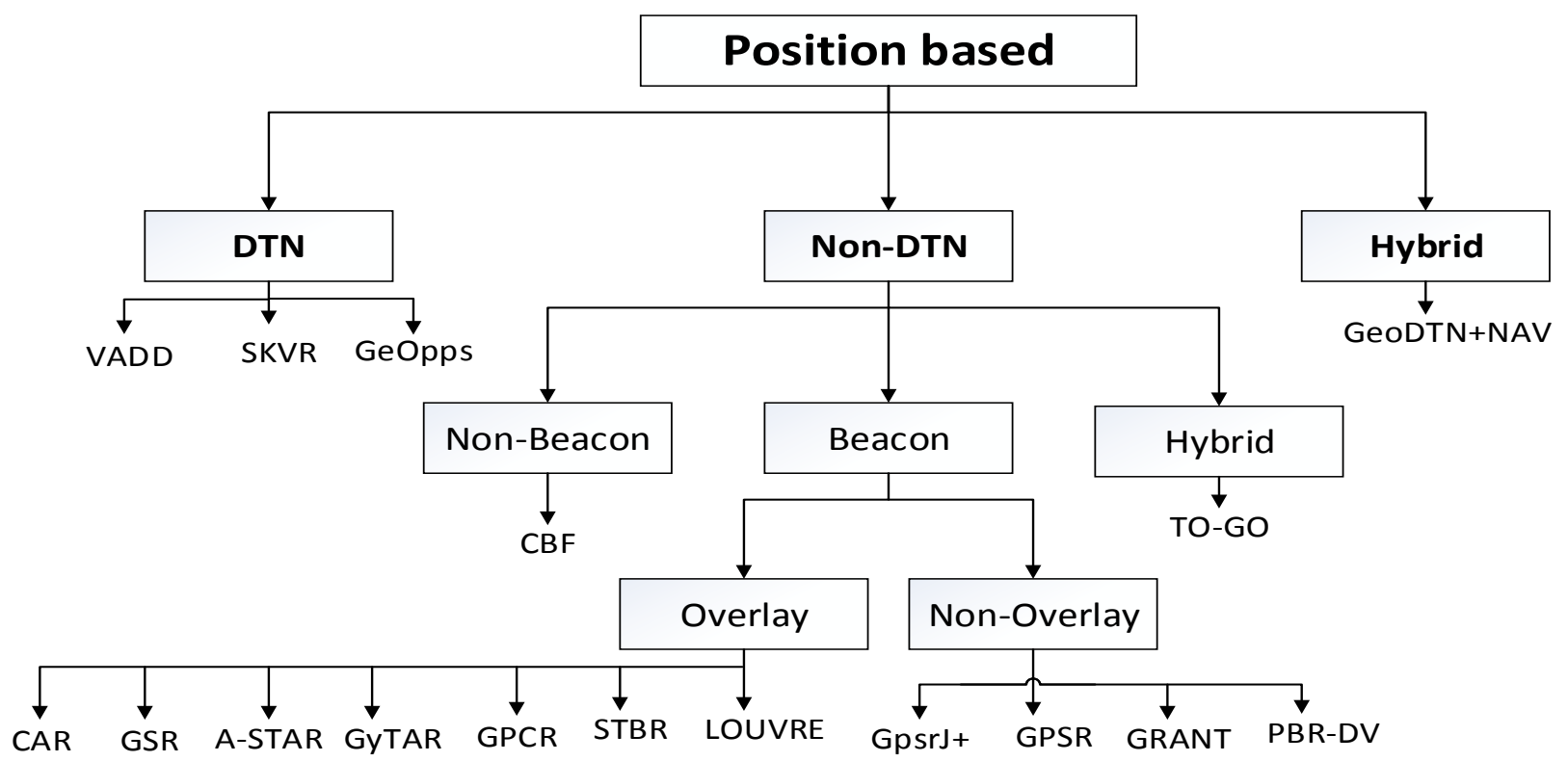

Figure 1 . Classification Of Position Based Routing Protocols in VANET

\section{NON-DELAY TOLERANT NETWORK (Non-DTN)}

Non DTN type of routing protocols [2] are very useful in highly congested areas. When forwarding strategy fails and local maximum occur then Non DTN type of routing protocol are used to recover from such failure. They are further divided as Beacon, non-Beacon and Hybrid.

\subsection{Non-beacon}

In non-beacon type of routing protocol, no beacon messages are transmitted but instead data packets are sent to direct neighbor. These neighbor then takes forwarding decision . e.g. contention based forwarding

\subsubsection{Contention Based Forwarding (CBF)}

In $\mathrm{CBF}$, [6] no beacon messages are used instead, greedy forwarding is used to route packet. In this protocol, next hop selection process is carried out in which all suitable nodes of forwarding node participate. Actual position of nodes plays vital role to take forwarding decision when packet is forwarded.

\subsection{Beacon}

In this type of routing, [2] beacon messages are used to gather information about neighbor nodes. It performs crucial role in routing process as well as forwarding process. They are further divided into two categories, i.e. overlay and non overlay.

\subsubsection{Overlay Routing}

In overlay routing, routing protocol works on the set of specific nodes which are overlaid on the top of current network. It has special mechanism to choose route at junction.

\subsubsection{Connectivity Aware Routing (CAR)}

It is proposed by Valery Neumov and Thomas R. Gross. The main feature of CAR [7] is that it not only locate destinations but also discover the paths between source and destination pairs. Its operation is divided into four main parts : destination location and path discovery, data packet forwarding along the discovered path, path maintenance with the help of guard and error recovery. CAR is based on two basic processes such as Preferred Group broadcasting (PGB)and Advanced Greedy
Forwarding (AGF) which tends to reduce overhead during routing. Each node periodically broadcast Hello Beacons along with its velocity vector information. Then the node that receives this Hello Beacon mark entry in its table. Destination location and also appropriate path to destination is discovered by means of PGB. If the path is not valid then guards are used for maintenance of path. If there is communication gap between the nodes then routing error occurs and these error are rectified by error recovery process.

\subsubsection{Geographic Source Routing(GSR)}

GSR protocol works by using neighboring node's location information [8] . Reactive Location Service (RLS) is used to find information associated with targeted node's location. In this protocol querying node broadcast 'position request' message in order to identify particular node. The requested node after receiving this message send 'position reply' message to querying node. By using digital map source node $S$ identifies location of all junction from $S$ to $D$ i.e. targeted location. Then by using Dijkstra algorithm shortest path from $S$ to endpoints are discovered. Then packet travels through GSR anchors i.e. junctions located in between shortest path to reach targeted location. These data packet contains location information of S, D and GSR junctions. GSR is independent of density of vehicle on the road while selecting route from $S$ to $\mathrm{D}$.

\subsubsection{Anchor based Street and Traffic Aware Routing (ASTAR)}

It depends on intensity of vehicles on the road .It contains two types of maps[9] : statistically rated map and dynamically rated map. Statistically rated map is generated based on stable amount of traffic on the road and dynamically rated graph is produced based on real time traffic condition on road. With the help of these maps and Dijkstra algorithm, anchor paths can be discovered. By using greedy forwarding, packets are transmitted between two adjacent nodes. When greedy forwarding fails and local optimum occurs, packets are recovered by using different algorithms. In this recovery algorithm, street is marked as "out of service" where local optimum occurs and this information is flooded in the network . By using this flooded information nodes come to 
know about failure path, which helps them to take better routing decision.

\subsubsection{Greedy Traffic Aware Routing (GyTAR)}

It is a junction based routing protocol. It employs greedy forwarding method [10] to forward packets to best junction. Carry and forward strategy is used to recover when local maximum occurs. In order to find positions of neighbor junction's digital map is used. A score of neighboring junction is calculated based on traffic density and distance of junction to destination.

\subsubsection{Greedy Perimeter Coordinator Routing (GPCR)}

It is proposed by Lochert et al .In this protocol, it is found that node planarization can be completely removed [11] as urban street maps naturally generate planar graphs. In planar graphs, node can forward as much as they can in both greedy and perimeter mode and cease their transmission at junctions where next road to turn has to be decide. GPCR rely on two heuristic to find out whether node is located at a junction. The first heuristic sends beacon messages to determine nodes location at junction. Second heuristic is obtained from correlation coefficient and if this correlation coefficient is closed to zero then node is located at junction . GPCR improves routing performance by removing node planarization.

\subsubsection{Street Topology Based Routing (STBR)}

In this protocol [12], randomly any node at junction is marked as master .Master checks whether links to next node are up or down. As every master knows link information of every other master thus, every master contains two level junction neighbor tables. The first level is from neighboring links to its direct junction nodes and second level is its direct junction nodes. It is from neighboring links to their own junction nodes. In STBR, geographic distance is used to route packet.

\subsubsection{Landmark Overlays for Urban Vehicular Routing Environment (LOUVRE)}

It comes under the category of geo-proactive overlay routing. In this, [13] overlay links remains connected even if vehicle density goes above threshold. Thus, because of this fact while developing overlay routes, many routes will use same overlay links. Due to this, while developing overlay routes delay is reduced. The main feature of this protocol is that vehicle density is determined in peer to peer fashion which tends to remove dependency on Road Side Units( RSU). Thus each vehicle knows the density of other connected roads in the network. Finally Dijkstra is used to find shortest path.

\subsubsection{Non-Overlay Routing}

In this routing, [2] first greedy forwarding is used to forward packet. In case if greedy forwarding fails and local maximum occurs then these protocols employs different recovery methods to recover from failure.

\subsubsection{Greedy Perimeter Stateless Routing at Junction $+($ GpsrJ+)}

It is proposed by Lee et al [14] .It is a position based routing protocol in which packet is first forwarded in greedy mode. In greedy mode packet is always send to a node which is closer to the destination. When greedy forwarding fails and packet reaches local maximum i.e. point at which no other node is closer to destination than the node itself then, node uses GpsrJ+ recovery mode. In this recovery mode, GpsrJ+ uses digital maps and packets are greedily moved in reverse direction along the perimeter of the roads. In order to find correct junction turns, it uses two hop neighbor information and thus determines correct routing paths. In GpsrJ+, if the node is having junction node as its neighbor then this junction node will forward packet and bypass it if possible. By using digital map and neighbor's location information, road segment onto which neighbor nodes are present is calculated. At last nodes place this information into beacon and flooded it to the forwarding nodes. GpsrJ+ can be used to improve the performance of GPCR and GPSR.

\subsubsection{Greedy perimeter Stateless Routing (GPSR)}

In GPSR [15] , two modes are used to forward a packet to the destination. First one is Greedy mode and second one is Perimeter mode. In greedy mode of forwarding, every node forwards a packet to a node which is geographically closer to the destination. Here every node need to know its own position, position of its neighbors and destination location .In VANET, GPS is used to know node's position, beacon messages are used to know neighbor node's position and destination location is obtained from separate location service. When this greedy forwarding fails and node reaches local maximum i.e. the point at which no other node is closer to destination than the node itself then GPSR forward packet by using perimeter mode. In perimeter mode, packet is forwarded by using right hand rule. While using right hand rule it is necessary that planar graph should not have any crossing edges. Thus, Relative Neighborhood Graph(RNG) or Gabriel Graph(GG)is used to obtain a network graph which is free of crossing edges.

\subsubsection{Greedy Routing with Abstract Neighbor Table (GRANT)}

It is proposed by Schnaufer in 2008 [12]. The main feature of GRANT is that, it contains Abstract Neighbor Table (ANT) by which planes are divided into separate areas and also there is only one representative neighbor per area. Abstract Neighbor Table (ANT) maintains entry of representative neighbor of each area and an empty entry is marked for areas which do not have neighbors. The concept of extended greedy routing is employed by GRANT, by which every node find its ' $x$ ' hop neighbors. This information helps to every node in choosing best route by avoiding local maximum. Let $\mathrm{E}$ be next forwarding neighbor, $\mathrm{D}$ be the destination and $\mathrm{N}$ be any node which is ' $\mathrm{x}$ ' hop away from $\mathrm{E}$. The metric for choosing next forwarding neighbor $\mathrm{E}$ depends upon multiplication of distance between node $\mathrm{N}$ and $\mathrm{D}$, minimal path from $\mathrm{N}$ to $\mathrm{E}$ and also charge per hop for multi hop neighbor. Next hop is selected based on smallest metric offered by neighbor $E$. These ' $\mathrm{x}$ ' hop neighbors are transmitted in beacon which increases overhead thus ANT is used to minimize it. As compared to greedy routing, path length of routes and packet recovery rate is less in GRANT.

\subsubsection{Position Based Routing with Distance Vector Recovery $(P B R-D V)$}

Basically, this protocol [12] is a combination of greedy routing and Ad-Hoc On-Demand Distance Vector Routing (AODV) recovery. This protocol uses Greedy mode and distance vector mode for forwarding a packet. If greedy mode fails then this protocol forwards packet by using Distance vector mode. In distance vector mode, node at local maximum broadcasts route request packet which contains its own id, location id and location of destination. Node which receives this request packet first computes its distance to destination and if its distance is more than the node from which it 
receives a request packet then it rebroadcast this packet and store sender node's id in routing table. Otherwise, it sends route reply packet to sender node. This sender node makes proper entry into its routing table in order to forward this reply packet to all the previous nodes from which it receives the request packet. When the reply packet is sent back to the node at local maximum, all the intermediate nodes make corresponding entry into routing table about previous nodes from which it receives reply packet and this information helps node at local maximum to reach a node which is closer to the destination. Once a packet reaches a node closer to the destination, again Greedy mode is used to forward the packet.

\subsection{Non-DTN Hybrid Routing}

It is a position based routing in which two-hop beaconing [2] is used to obtain topology knowledge and then it exploit this knowledge to choose appropriate target forwarder. A target node is the node which is not located at the junction.

e.g. TO-GO

\subsubsection{Topology-assist Geo-Opportunistic Routing (TO-GO)}

It is a geographical routing protocol in which Next hop Prediction Algorithm(NPA)[16] is used to mark the packet for target node and then this packet is broadcasted. This packet will be received by nodes at target node or by the nodes which are closer to the target node. Then the nodes in forwarding set selection (FSS), set their timer by estimating their distance to target node. Enhanced beacon is used to determine the target node, it can be furthest node or can be junction node. Right hand rule is used to determine best forwarding nodes and best directions. If the direction of furthest node is same as that of best direction then best forwarding node is the furthest node otherwise junction node is best forwarding node. After finding FSS, timer is set such that the node closer to target node must be the next forwarder. Once the packet is received by the destination node, destination node informs all of its neighbor about packet arrival so that packet duplication is avoided.

\section{HYBRID ROUTING PROTOCOL}

Hybrid routing protocol is a position based routing protocol which work by combining the best characteristic of DTN and Non-DTN routing protocols to recover from failure of greedy as well as recovery mode.

\subsection{Geographic and DTN Routing with Navigation Assistance (GeoDTN+Nav)}

It is a geographic routing protocol which forwards the packet [17] by using three modes such as greedy mode, Perimeter mode and DTN mode. In greedy mode, packet is always forwarded to the node which is geographically closer to the destination. But because of obstacle greedy forwarding can be fail and local maximum occurs i.e. no other node is closer to the destination than the sender node itself. Then this protocol recover by using perimeter mode in which packet is recovered from the local maximum by using planarization and again switched to greedy mode. But because of highly dynamic nature of VANET, it is not necessary that network will be connected at all the times. Network may get disconnected or partitioned. Greedy mode and Perimeter mode cannot work efficiently in case of partition network. Thus DTN mode is used to successfully deliver packet in case of disconnected network. DTN mode uses mobility of nodes in the VANET to reach destination. Depending on network disconnections and delivery quality of the node which stores packet, node is switched to DTN mode.

\section{CONCLUSION}

Routing is the most important scheme by which data packets are transmitted from source to the destination. For highly dynamic and infrastructure less network like VANET, it is very important to apply correct routing protocol depending on the type of application. As discussed in the paper, each position based routing protocol is having different characteristic .These characteristics helps to choose correct routing protocol for a particular application. As in case of position based routing protocol, each node is aware of its own location and its neighbor node location, thus they are highly suitable for VANET. In this paper, the survey of various position based routing protocols has been done.

The performance of any routing protocol depends on parameters like delay, throughput, routing overhead etc. Swarm intelligence is an emerging concept in the field of VANET. There are various optimization techniques that come under swarm intelligence. These optimization techniques help to achieve best probable results under the given conditions. Thus in future work, by using these optimization techniques the performance of the position based routing protocols can be improved.

\section{REFERENCES}

[1] Saif Al-Sultan, Moath M. Al-Doori, Ali H. Al-Bayatti, Hussein Zedan, "A comprehensive survey on vehicular ad hoc network", Journal of Network and Computer Applications, vol. 37, 2014, pp. 380-392.

[2] Mostofa Kamal Nasir, Mohammad Khaled Sohel, Mohammad Touhidur Rahman, A.K.M. Kamrul Islam “ A Review on Position Based Routing Protocol in Vehicular Adhoc Network" , American Journal of Engineering Research (AJER), Volume-02, , 2013, pp $7-13$

[3] J.Zhao, G.Cao," VADD: vehicle-assisted data delivery in vehicular ad hoc networks", in Proceedings of INFOCOM, 2006, pp.1-12.

[4] Ahmed S. Kanere "SKVR: scalable knowledge-based routing architecture for public transport networks", In Proceedings of the 3rd international workshop on Vehicular ad hoc networks, 2006, pp. 92-93.

[5] Leontiadis I. Mascolo, C "Geopps: Geographical opportunistic routing for vehicular networks", IEEE International Symposium on a, 2007, pp. 1-6.

[6] H. Fiifler, H. Hartenstein, Widmer, M. Mauve and W. Effelsberg, "Contention-Based Forwarding for Street Scenarios" , In Proc. of the 1st International Workshop on Intelligent Transportation (WIT), Hamburg, Germany, March 2004, pp. 155-160.

[7] V. Naumov, T. R.Gross, "Connectivity Aware Routing In Vehicular ad hoc network", in Proceedings of INFOCOM, 2007, pp.1-9.

[8] Lichuan Liu, Zhigang Wang, Wern-Kueir Jehng "A Geographic Source Routing Protocol for Traffic Sensing in Urban Environment" ,4th IEEE Conference on Automation Science and Engineering , IEEE Publications, 2008August 23-26.

[9] B. C. Seet; et al., "A-STAR: A mobile ad hoc routing strategy for metropolis vehicular communications," Proc. networking 2004, pp. 989-999. 
[10] Moez Jerbi, Rabah Meraihi, Sidi Mohammed Senouci, and Yacine Ghamri Doudane "GyTAR: improved Greedy Traffic Aware Routing Protocol for Vehicular Ad Hoc Networks in City Environments",

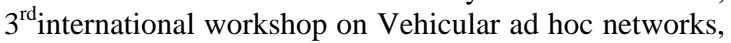
2006, pp. 88-89.

[11] Jerbi, M. Senouci, S. M. Meraihi, R. Ghamri-Doudane "An improved vehicular ad hoc routing protocol for city environments", ICC'07, 2007, pp. 3972-3979.

[12] Kevin C. Lee, Uichin Lee, Mario Gerla, "Survey of Routing Protocols in Vehicular Ad Hoc Networks", car 2 car communication, consortium

[13] Lee, K., Le, M., Haerri J., and Gerla, M,"Louvre: Landmark overlays for urban vehicular routing environments," Proceedings of IEEE WiVeC, 2008.
[14] K. C. Lee, et al., "Enhanced Perimeter Routing for Geographic Forwarding Protocols in Urban Vehicular Scenarios," in IEEE Globecom Workshops, pp. 1-10, 2007.

[15] Darshana , Sandeep Mann "Greedy Perimeter Stateless Routing In Vehicular AdHoc Networks", IJAEST, Volume 2, pp 74-79

[16] K. C. Lee, et al., "TO-GO: Topology-assist geoopportunistic routing in urban vehicular grids," in Sixth International Conference on Wireless On-Demand Network Systems and Services (WONS 2009). pp. 1118, 2009.

[17] P.-C. Cheng, et al., "GeoDTN+Nav: Geographic DTN Routing with Navigator Prediction for Urban Vehicular Environments," Mob. Netw. Appl., vol. 15, pp 61-82 ,2010. 